\title{
Evidencias de Validez de la Versión Española de las Sexual Inhibition/Sexual Excitation Scales-Short Form (SIS/SES-SF)
}

\author{
Validity Evidences of the Spanish Version of the Sexual Inhibition/Sexual Excitation \\ Scales-Short Form (SIS/SES-SF)
}

\author{
Juan Carlos Sierra ${ }^{1}$, María Dolores de la Rosa Centella ${ }^{1}$, Reina Granados ${ }^{1}$, Cristóbal Calvillo ${ }^{1}$, Ana \\ Isabel Arcos-Romero ${ }^{1}$, María del Mar Sánchez-Fuentes ${ }^{2}$ y Nieves Moyano ${ }^{3}$
}

\begin{abstract}
Resumen
Se examinó la validez de la versión española de las Sexual Inhibition/Sexual Excitation Scales-Short Form (SIS/SES-SF), relacionándo las con excitación subjetiva y respuesta genital ante estímulos sexuales. Una muestra de 164 jóvenes heterosexuales completó el SIS/SES-SF y el MGH-SFQ. Después realizaron una tarea experimental consistente en la visualización de un vídeo de contenido neutro y un video con contenido sexual explícito, mientras se evaluaba la respuesta genital y la excitación subjetiva. Los resultados mostraron diferencias significativas entre el vídeo neutro y el erótico en la excitación objetiva y subjetiva en ambos sexos. Se obtuvieron correlaciones significativas entre SES y excitación subjetiva en mujeres. La propensión a excitarse explicó el $18 \%$ de la varianza de la excitación subjetiva y el $6 \%$ de las sensaciones genitales femeninas. La inhibición sexual relacionada con el miedo a la ejecución sexual tuvo capacidad para discriminar dificultades en el funcionamiento sexual en ambos sexos. Finalmente, se avala la necesidad de evaluar conjuntamente la excitación subjetiva y la objetiva.
\end{abstract}

Palabras clave: Sexual Inhibition/Sexual Excitation Scales-Short Form (SIS/SES-SF), excitación sexual subjetiva, respuesta genital, validez predictiva

\begin{abstract}
Predictive validity of the Spanish version of the Sexual Inhibition/Sexual Excitation Scales-Short Form (SIS/SES-SF) was examined. We correlated sexual inhibition and sexual excitation with subjective arousal and genital response during exposure to visual erotic stimuli. A sample of 164 heterosexual young people completed the SIS/SES-SF and the MGH-SFQ. Participants performed an experimental sequence in which a neutral content film and an erotic film were presented. In the meantime, genital response and subjective sexual arousal were evaluated. Results showed significant differences between neutral and sexually explicit films in objective and subjective arousal in both men and women. A significant correlation between SES and subjective arousal was obtained in women. Propensity to become aroused explained $18 \%$ of the variance of female subjective arousal and $6 \%$ of female genital sensations. Sexual inhibition related to the threat of sexual performance failure was able to discriminate difficulties in sexual functioning in both men and women. Finally, there is a need to assess the subjective sexual arousal along with the objective sexual response.
\end{abstract}

Keywords: Sexual Inhibition/Sexual Excitation Scales-Short Form (SIS/SES-SF), subjective sexual arousal, genital response, predictive validity

Financiación: este estudio fue financiado gracias a los Proyectos de Investigación PSI2010-15719 (Ministerio de Ciencia e Innovación) y PSI2014-25035-R (Ministerio de Economía y Competitividad).

\footnotetext{
${ }^{1}$ Centro de Investigación Mente, Cerebro y Comportamiento (CIMCYC), Universidad de Granada, España.

${ }^{2}$ Facultad de Ciencias Humanas y Sociales, Departamento de Psicología del Individuo, Universidad de la Costa (CUC), Barranquilla, Colombia.

${ }^{3}$ Facultad de Ciencias Humanas y de la Educación, Departamento de Psicología y Sociología, Universidad de Zaragoza, Huesca, España

Correspondencia: Juan Carlos Sierra. Facultad de Psicología. Campus Universitario de Cartuja, s/n. 18011. Granada, España. Correo: jcsierra@ugr.es
}

Revista Iberoamericana de Diagnóstico y Evaluación - e Avaliação Psicológica. RIDEP · No50 · Vol.1 · 173-184 · 2019

ISSN: 1135-3848 print /2183-6051online 


\section{Introducción}

El Modelo de Control Dual (MCD) de la respuesta sexual plantea la existencia de un sistema excitativo y otro inhibitorio, relativamente independientes, que al actuar de forma conjunta proporcionan un "doble control" sobre la respuesta sexual y, consecuentemente, sobre el comportamiento sexual en hombres y en mujeres (Bancroft, 1999; Bancroft, Graham, Janssen, \& Sanders, 2009). En este sentido, la excitación sexual $^{l}$ sería producto del balance entre ambos sistemas. Un principio básico del MCD es la variabilidad individual, según la cual los individuos difieren en su propensión hacia la inhibición o excitación sexuales. Este modelo teórico asume que la inhibición sexual actúa de forma adaptativa en situaciones amenazantes, ayudando a mantener al individuo fuera de problemas (Bancroft, 1999; Bancroft \& Janssen, 2000). De la misma forma, la activación de estos dos sistemas da lugar a la excitación sexual que resultará adaptativa 0 , por el contrario, disfuncional (Janssen \& Bancroft, 2007). Así, niveles elevados de inhibición sexual se han asociado con mayor vulnerabilidad a desarrollar disfunciones sexuales, como problemas de erección (Bancroft, Carnes, Janssen, Goodrich, \& Long, 2005; Bancroft \& Janssen, 2000; Moyano \& Sierra, 2014), eyaculación precoz o bajo deseo sexual (Bancroft, 1999; Bancroft, Herbenick et al., 2005; Prause, Janssen, \& Hetrick, 2008; VallejoMedina, Pérez-Durán, \& Saavedra-Roa, 2018).

Con el fin de evaluar la propensión hacia la inhibición/excitación sexual se han desarrollado diversas escalas basadas en el MCD. Las primeras fueron las Sexual Inhibition/Sexual Excitation Scales (SIS/SES), desarrolladas en hombres (Janssen, Vorst, Finn, \& Bancroft, 2002a) y posteriormente validadas en mujeres (Carpenter, Janssen, Graham, Vorst, \& Wicherts, 2008). Debido a que no todos sus ítems resultaban igual de relevantes para hombres y mujeres (Carpenter et al., 2008), se elaboró una nueva versión reducida equivalente para ambos sexos (SIS/SES-

\footnotetext{
${ }^{1}$ Con el objeto de evitar confusiones, la excitación sexual referida a uno de los componentes de la respuesta sexual aparecerá en cursiva para diferenciarla de la excitación sexual como componente del MCD.
}

SF; Carpenter, Janssen, Graham, Vorst, \& Wicherts, 2011), la cual resulta de una selección de los ítems de las SIS/SES que mejor representan la estructura del MCD en hombres y en mujeres (Carpenter et al., 2008; Janssen et al., 2002a).

Las escalas SIS/SES-SF, validadas en población española por Moyano y Sierra (2014), incluyen 14 ítems distribuidos en tres subescalas: (1) Excitación Sexual (SES, por sus siglas en inglés) que evalúa la excitación sexual derivada de interacciones sociales (e. g., "Cuando un/a desconocido/a sexualmente atractivo/a me toca accidentalmente, me excito con facilidad"), (2) Inhibición Sexual-1 (SIS1, por sus iniciales en inglés), que incluye ítems relacionados con la inhibición sexual por miedo al rendimiento sexual, referidos a la distracción/concentración en la ejecución sexual (e. g., "No consigo excitarme a menos que me concentre exclusivamente en la estimulación sexual"); y (3) Inhibición Sexual-2 (SIS2, por sus iniciales en inglés), referida a la inhibición sexual por miedo a las consecuencias de la ejecución sexual, es decir, miedo al riesgo de ser descubierto o contraer algún tipo de infección de transmisión sexual (e. g., "Si creo que otras personas pudieran verme mientras tengo relaciones sexuales, es poco probable que mantuviese la excitación"). Sus puntuaciones correlacionaron con la versión extensa (Janssen et al., 2002a) de 45 ítems en hombres: SES ( $r=.90)$, SIS1 $(r=.80)$ y SIS2 $(r=.80)$. Además, mostró coeficientes aceptables de fiabilidad test-retest: entre .61 de SES y SIS1 en mujeres, y .75 de SES en hombres (Carpenter et al., 2011). La adaptación española de las SIS/SES-SF presentó una estructura similar a la versión original, con equivalencia factorial entre hombres y mujeres. Sus coeficientes de fiabilidad de consistencia interna fueron aceptables, oscilando entre .60 (SIS2) y .72 (SES) en hombres, y entre .62 (SIS2) y .71 (SES) en mujeres. Además, sus puntuaciones correlacionaron en el sentido esperado con diferentes dimensiones de la respuesta sexual (deseo sexual, excitación y orgasmo). En cuanto a las diferencias entre sexos, los hombres obtuvieron puntuaciones más elevadas en SES mientras que las mujeres lo hicieron en SIS1 (Moyano \& Sierra, 2014).

La relación entre las dimensiones excitación e inhibición sexuales planteadas por el MCD y la 
excitación sexual genital y subjetiva ante estímulos sexuales ha sido estudiada tanto en hombres como en mujeres. Janssen, Vorst, Finn, y Bancroft, (2002b) observaron que hombres con alto rasgo excitativo mostraban mayor excitación sexual, tanto subjetiva como genital. En cuanto a las mujeres, mayor propensión para excitarse se relacionó con mayor excitación sexual subjetiva (e. g., Gregory, Cheng, Rupp, Sengelaub, \& Heiman, 2015; Landry, 2016; Macapagal, Janssen, Fridberg, Finn, \& Heiman, 2011; Rupp et al., 2009). Por otro lado, la acción conjunta de la excitación e inhibición sexuales predijo de forma significativa la respuesta genital en mujeres (Velten, Scholten, Graham, \& Margraf, 2017). Por tanto, las escalas SIS/SES-SF constituyen un instrumento sencillo que puede ayudar a predecir el nivel de excitación sexual estado en una situación concreta $\mathrm{y}$, consecuentemente, la presencia de dificultades que caracterizarían a individuos con disfunciones sexuales que afectan a la calidad de vida (Latorre Román, López Munera, Izquierdo Rus, \& García Pinillos, 2018; López Huerta, González Romo, \& Tejada Tayabas, 2017).

El objetivo general del presente estudio es aportar evidencias de validez a las medidas de la versión española de las SIS/SES-SF. El primer objetivo específico es examinar la relación de las dimensiones excitación e inhibición sexuales con la excitación sexual subjetiva y objetiva (respuesta genital) ante estímulos sexuales visuales. El segundo objetivo específico es analizar la capacidad de las SIS/SES-SF para diferenciar a personas con y sin dificultades en la fase de excitación de la respuesta sexual. A este respecto, en primer lugar, se espera que la propensión a excitarse se asocie con mayor excitación sexual subjetiva y con mayor intensidad de la respuesta genital ante estímulos visuales (Granados, Carvalho, \& Sierra, 2018; Hodgson, 2013; Hodgson, Kukkonen, Binik, \& Carrier, 2016; Janssen et al., 2002b), mientras que la propensión a inhibirse se asociará con una menor excitación sexual subjetiva y objetiva ante estímulos visuales (Hodgson et al., 2016). Asimismo, se espera que los participantes con más dificultades en la fase de excitación de la respuesta sexual muestren menor propensión a excitarse y mayor propensión a inhibirse que aquellos que informen de un funcionamiento sexual normal (Moyano \& Sierra, 2014; Santos-Iglesias, Castro, Barbosa, \& Nobre, 2017).

\section{Método}

\section{Participantes}

Mediante un muestreo incidental se seleccionaron 164 jóvenes heterosexuales españoles (69 hombres y 95 mujeres), con edades comprendidas entre 18 y 25 años. Se consideraron como criterios de exclusión padecer alguna enfermedad física, trastorno psicológico o disfunción sexual, o informar de abuso de alcohol o consumo de sustancias/fármacos que pudiesen afectar a la respuesta sexual. Todos los participantes eran estudiantes universitarios de diversas facultades de la [Universidad de Granada]. En la Tabla 1 se presentan las principales características sociodemográficas de la muestra.

Tabla 1. Características sociodemográficas de la muestra

\begin{tabular}{lcc}
\hline & $\begin{array}{c}\text { Hombres } \\
(n=69)\end{array}$ & $\begin{array}{c}\text { Mujeres } \\
(n=95)\end{array}$ \\
\hline $\begin{array}{l}\text { Edad } \\
\text { Media }(D T)\end{array}$ & $20.55(1.68)$ & $20.49(1.75)$ \\
$\begin{array}{l}\text { Edad de la primera } \\
\text { relación sexual }\end{array}$ & & \\
$\begin{array}{l}\text { Media (DT) } \\
\text { Relación de pareja en la } \\
\text { actualidad }\end{array}$ & $16.86(1.45)$ & $16.17(1.30)$ \\
Sí & & \\
No & $35.8 \%$ & $71.3 \%$ \\
$\begin{array}{l}\text { Número de parejas } \\
\text { sexuales }(D T)\end{array}$ & $64.2 \%$ & $28.7 \%$ \\
& $4.47(3.97)$ & $4.12(3.48)$ \\
\hline
\end{tabular}

\section{Instrumentos y materiales}

\section{Cuestionario Sociodemográfico y de la Historia}

Sexual. Incluye preguntas sobre sexo, edad, orientación sexual, edad de la primera relación sexual, relación de pareja y número de parejas sexuales. Además, pregunta acerca de problemas médicos y psicológicos, tratamientos (médico y/o psicológico) y consumo de alcohol, sustancias o fármacos.

$\begin{array}{cccc}\text { Versión española } & \text { de las } & \text { Sexual } \\ \text { Inhibition/Excitation } & \text { Scales-Short } & \text { Form }\end{array}$
(SIS/SES-SF; Carpenter et al., 2011) de Moyano y Sierra (2014). Consta de 14 ítems que se contestan en una escala tipo Likert con cuatro opciones de 
respuesta desde 1 (completamente de acuerdo) a 4 (completamente en desacuerdo). Puntuaciones elevadas indican mayor excitación o mayor inhibición sexual. En el presente estudio, la fiabilidad en la muestra de hombres fue de .74, .62 y .59 en SES, SIS1 y SIS2, respectivamente; en mujeres, fue .80 en SES, .65 en SIS1 y .69 en SIS2.

Versiones españolas de las escalas de Valoración de Excitación Sexual (VES; Mosher, 2011) y Valoración de Sensaciones Genitales (VSG; Mosher, 2011) de Sierra et al. (2017). La VES consta de cinco ítems: (1) nivel global de excitación sexual, (2) intensidad de las sensaciones genitales, (3) sensación de calor experimentada, (4) sensaciones físicas no genitales y (5) nivel de concentración sexual. Utiliza una escala tipo Likert de siete puntos desde 1 (ninguna excitación) a 7 (extremadamente excitado/a). Su fiabilidad de consistencia interna es de .90. En este estudio, se obtuvo un alfa de Cronbach de .90 en hombres y de .88 en mujeres. Por su parte, la VSG está formada por una lista de 11 descripciones de las sensaciones genitales, que van incrementando su intensidad desde 1 (ninguna sensación genital) a 11 (orgasmo múltiple). Se debe seleccionar la opción que mejor refleje el máximo nivel de sensaciones genitales experimentadas en un momento dado, por ejemplo, durante la exposición a un estímulo erótico. En la adaptación española, sus puntuaciones correlacionaron con las de la escala VES ( $r=.73$ ). En la muestra de este estudio, las correlaciones entre VES y VSG fueron .78 en hombres y .67 en mujeres. En ambas escalas, mayor puntuación indica mayor excitación sexual subjetiva.

Versión española del Massachusetts General Hospital-Sexual Functioning Questionnaire (MGH-SFQ; Fava, Rankin, Alpert, Nierenberg, \& Worthington, 1998) de Sierra, Vallejo-Medina, Santos Iglesias y Lameiras Fernández (2012). Incluye cinco ítems que evalúan deseo sexual, excitación, erección (en hombres), orgasmo y satisfacción sexual, con relación al último mes (e. g., “¿Cómo ha sido su deseo sexual?”; “¿Con qué facilidad ha alcanzado un orgasmo?"). Utiliza una escala tipo Likert de 5 puntos que van desde 0 (totalmente disminuido/a) a 4 (normal). Mayor puntuación indica mejor funcionamiento sexual.
Sierra et al. (2012) informaron valores de consistencia interna de .90 y .93 en hombres y mujeres, respectivamente, así como buenos indicadores de validez de sus medidas. En el presente estudio se empleó únicamente el ítem 2 (excitación).

Excitación sexual objetiva: respuesta genital. Para registrar la respuesta genital en los hombres se empleó un pletismógrafo (Janssen, Vissenberg, Visser, \& Everaerd, 1997), el cual registra los cambios en la circunferencia del pene resultado de la erección. La evaluación de la respuesta genital en las mujeres se realizó mediante fotopletismografía vaginal (Sintchak \& Geer, 1975) obteniéndose como medida la amplitud de pulso vaginal (Laan, Everaerd, \& Evers, 1995). Se utilizó el sistema Biopac MP150 y el software Acknowledge 4.2.0 (BIOPAC Systems, Inc., Goleta, CA, USA) para obtener y procesar los datos registrados. Las respuestas sexuales fisiológicas se transformaron en puntuaciones $z$ con el fin de comparar las puntuaciones en ambos sexos. Mayor puntuación indica mayor respuesta fisiológica. A partir de las puntuaciones $z$ obtenidas, se distinguió en hombres y mujeres "bajos", si las puntuaciones se ubicaban por debajo de la media 0 , y "altos" si las puntuaciones superaban el valor 0 . Las respuestas genitales se definieron en términos de diferencias entre la línea de base y el estímulo sexual.

Estímulos sexuales visuales. Se utilizaron dos vídeos de tres minutos de duración cada uno. El primero de ellos fue un vídeo de contenido neutro (documental sobre la naturaleza) que se utilizó como medida de línea base. El segundo fue de contenido sexual explícito en el que una pareja heterosexual mantiene relaciones sexuales, incluyendo sexo oral y coito vaginal. Se utilizó un vídeo distinto para hombres y mujeres, como resultado de la validación previa que se hizo de los mismos (Sierra, Granados, Sánchez-Fuentes, Moyano, \& López, 2015).

\section{Procedimiento}

Las personas interesadas en participar en el estudio aceptaron en formato online un consentimiento informado donde se explicaba el objetivo del estudio y la tarea a realizar, y contestaron el Cuestionario Sociodemográfico y de la Historia Sexual, las escalas SIS/SES-SF y el 
MGH-SFQ. Con el objeto de no usar datos de carácter identificativo, al comienzo del estudio los participantes proporcionaron un código (inicial del primer nombre, día y mes de nacimiento) con el que se les reconoció durante todas las fases del estudio. A aquellos participantes que cumplían los criterios de inclusión, se les contactó por vía telefónica para continuar en la siguiente etapa del estudio. Se les explicó el objetivo de la investigación y fueron citados para acudir al laboratorio. Asimismo, se pidió a los participantes abstenerse de consumir alcohol, cafeína y de realizar actividades sexuales en las 24 horas previas a los registros en el laboratorio de sexualidad con el fin de reducir al máximo las posibles fuentes fisiológicas que pudieran interferir en las respuestas (Bradford \& Meston, 2006). Del mismo modo, para evitar diferencias hormonales asociadas al ciclo menstrual, las mujeres fueron citadas entre los días 14 al 28 de su ciclo (Suschinsky, Bossio, \& Chivers, 2014). Una vez en el laboratorio, se les informaba nuevamente del objetivo y de la tarea a realizar en el procedimiento experimental, para lo cual el investigador (hombre para los participantes hombres y mujer para las participantes mujeres) mostraba los dispositivos de registro y explicaba su correcta colocación. El participante fue informado de que en cualquier momento podía abandonar el estudio. Por último, se aceptaba el consentimiento informado de esta segunda fase del estudio, en el que se garantizaba el anonimato, confidencialidad y voluntariedad en el mismo, sin recibir compensación de ningún tipo por la participación.

Una vez que los dispositivos de registro eran colocados de forma privada por el propio participante, este comunicaba su disposición para comenzar la sesión por medio de un intercomunicador. El participante permanecía sentado inmóvil frente a una pantalla, dejando transcurrir cinco minutos de adaptación y habituación a la sala experimental (insonorizada, y con temperatura y luminosidad constantes), así como a los instrumentos de registro. Una vez transcurrido este tiempo, comenzaba la tarea experimental, mostrándose en la pantalla los vídeos de contenido neutro y sexual explícito, con una duración de tres minutos cada uno de ellos. Al final de cada vídeo, los participantes contestaban las escalas VES y VSG, en formato papel y lápiz. Los vídeos se proyectaron en una pantalla LCD de 24" colocada a unos 100 centímetros del participante. El tiempo aproximado para realizar la tarea fue 15 minutos. El estudio fue previamente aprobado por el Comité Ético de Investigación Humana de la [Universidad de Granada].

\section{Resultados}

En primer lugar, se obtuvieron los estadísticos descriptivos de las variables evaluadas, y se examinaron las diferencias por sexo (véase la Tabla 2), encontrándose únicamente diferencias en SIS2. En segundo lugar, un ANOVA de medidas repetidas indicó diferencias significativas entre la exposición a estímulos visuales neutros y eróticos en hombres, tanto en VES [Lambda de Wilks $F(1,66)=257.56, p=.000, \eta^{2}=.79$ ] como en VSG [Lambda de Wilks $F(1,65)=175.11, p=.000$, $\left.\eta^{2}=.72\right]$, y en mujeres, igualmente en VES [Lambda de Wilks $F(1,93)=513.91, p=.000$, $\left.\eta^{2}=.84\right] \quad$ y VSG [Lambda de Wilks $F(1$, 89) $=320.35, p=.000, \eta^{2}=.78$ ], siendo superior la excitación sexual informada en VES y VSG ante los estímulos visuales de contenido erótico que ante los neutros. Asimismo, se comprobó si existían diferencias significativas en la excitación sexual objetiva ante ambos tipos de estímulos, y se encontró que esta fue mayor ante los eróticos que ante los neutros, tanto en hombres [Lambda de Wilks $F(1,67)=2522.03 ; p=.000] ; \quad \eta^{2}=.97$, como en mujeres [Lambda de Wilks $\left.F(1,94)=113.83 ; p=.000 ; \eta^{2}=.54\right]$. La excitación sexual objetiva, al comparar a partir de las puntuaciones $z$, no resultó significativamente diferente entre hombres y mujeres.

A continuación se realizaron dos análisis de regresión lineal con el fin de estimar la capacidad de SIS/SES-SF para predecir la excitación sexual subjetiva (VES y VSG). Previamente, a través de un análisis de correlaciones bivariadas, se observó si existían correlaciones estadísticamente significativas entre las puntuaciones del SIS/SES y las otras medidas. De este modo, aquellas variables que no mostraron tener asociaciones significativas (excitación sexual objetiva), no fueron consideradas para el posterior análisis de regresión.

A través del método "Introducir" se incluyeron 
Tabla 2. Estadísticos descriptivos de las variables evaluadas según sexo

\begin{tabular}{|c|c|c|c|c|c|}
\hline & \multicolumn{2}{|l|}{ Hombres } & \multicolumn{2}{|l|}{ Mujeres } & \multirow[b]{2}{*}{$t / \chi^{2}$} \\
\hline & Rango & $M(D T)$ & Rango & $M(D T)$ & \\
\hline SES & $11-13$ & $16.33(2.94)$ & $8-23$ & $15.41(3.17)$ & 1.89 \\
\hline SIS1 & $4-12$ & $8.03(2.05)$ & $4-14$ & $8.05(1.95)$ & -0.07 \\
\hline SIS2 & $5-16$ & $10.45(2.35)$ & $6-16$ & $11.96(2.24)$ & $-4.14 * * *$ \\
\hline VES neutro & $5-10$ & $6.08(1.56)$ & $5-17$ & $6.42(2.23)$ & -1.05 \\
\hline VES erótico & $5-31$ & $18.61(6.51)$ & $7-34$ & $19.72(5.69)$ & -0.38 \\
\hline VSG neutro & $1-2$ & $1.03(0.17)$ & $1-3$ & $1.04(.25)$ & -1.15 \\
\hline VSG erótico & $1-6$ & $3.33(1.40)$ & $1-7$ & $3.70(1.43)$ & -1.59 \\
\hline $\begin{array}{l}\text { Excitación objetiva } \\
\text { neutro }\end{array}$ & $71.37-153.88$ & $99.84(15.74)$ & $0.002-0.186$ & $0.049(0.040)$ & \\
\hline Puntuaciones $z$ & $-1.81-3.43$ & $-.0003(1.00)$ & $-0.75-8.14$ & $0.0081(1.00)$ & 0.59 \\
\hline Bajo (\%) & $39 \%$ & & $61 \%$ & & \\
\hline Alto (\%) & $45.2 \%$ & & $54.8 \%$ & & \\
\hline $\begin{array}{l}\text { Excitación objetiva } \\
\text { erótico }\end{array}$ & $69.72-160.74$ & $101.56(18.42)$ & $0.001-0.668$ & $0.058(-0.075)$ & \\
\hline Puntuaciones $z$ & $-1.73-3.21$ & $0.0001(1.00)$ & $-1.17-3.45$ & $0.018(1.00)$ & 0.00 \\
\hline Bajo (\%) & $42 \%$ & & $58 \%$ & & \\
\hline Alto (\%) & $41.3 \%$ & & $58.7 \%$ & & \\
\hline $\begin{array}{l}\text { Excitación objetiva } \\
\text { global }\end{array}$ & $0.74-39.32$ & $17.69(9.80)$ & $-0.58-0.20$ & $0.05(0.08)$ & \\
\hline Puntuaciones $z$ & $-1.65-2.88$ & $0.001(1.00)$ & $-7.79-1.93$ & $0.002(1.01)$ & 1.87 \\
\hline Bajo (\%) & $36.8 \%$ & & $63.2 \%$ & & \\
\hline Alto (\%) & $47.4 \%$ & & $47.4 \%$ & & \\
\hline
\end{tabular}

Nota. Rango: rango de las respuestas dadas, no de la escala. SES: excitación sexual. SIS1: inhibición sexual por miedo al fallo en el rendimiento/ejecución sexual; SIS2: inhibición sexual por miedo a las consecuencias de la ejecución sexual. Excitación objetiva global: promedio del grado de erección en los hombres y amplitud del pulso vaginal en las mujeres ante la visualización de estímulos neutros y eróticos. $p<.001$.

Tabla 3. Resumen del modelo de regresión con VES como variable dependiente en mujeres

\begin{tabular}{ccccc}
\hline & $B$ & EE & $\beta$ & $t$ \\
\hline SES & .87 & .19 & .40 & $4.61^{* * *}$ \\
SIS1 & -.09 & .32 & -.03 & -0.29 \\
SIS2 & .26 & .31 & .10 & 0.84 \\
\hline
\end{tabular}

Nota. SES: excitación sexual. SIS1: inhibición sexual por miedo al fallo en el rendimiento/ejecución sexual; SIS2: inhibición sexual por miedo a las consecuencias de la ejecución sexual. $* * * p<.001$.

Tabla 4. Resumen del modelo de regresión con VSG como variable dependiente en mujeres

\begin{tabular}{lcccc}
\hline & $B$ & EE & $\beta$ & $t$ \\
\hline SES & .13 & .05 & .28 & $2.44^{*}$ \\
SIS1 & -.13 & .09 & -.18 & -1.50 \\
SIS2 & .07 & .08 & .12 & 0.90 \\
\hline
\end{tabular}

Nota. SES: excitación sexual. SIS1: inhibición sexual por miedo al fallo en el rendimiento/ejecución sexual; SIS2: inhibición sexual por miedo a las consecuencias de la ejecución sexual. ${ }^{*} p<.05$.

en bloque las variables SES, SIS1 y SIS2 en un único paso. En hombres, los resultados no mostraron modelos significativos. En cambio, en las mujeres se obtuvo un modelo de regresión que explicó un $18 \%\left(R^{2}\right.$ ajustada $\left.=.18, p<.001\right)$ de la varianza de VES $[F(3,89)=7.70, p<.001]$ con SES como único predictor (Tabla 3). También se obtuvo un modelo significativo que explica el $6 \%$ $\left(R^{2}\right.$ ajustada $\left.=.06, p<.05\right)$ de la varianza de VSG $[F(3,85)=2.86, p<.05]$, con SES como único predictor.

Por último, mediante MANOVA se compararon, en hombres y mujeres, las puntuaciones SIS/SES-SF entre un grupo con dificultades en la capacidad de excitación sexual (puntuaciones de 0 a 3, es decir, desde totalmente disminuido a mínimamente disminuido, en el ítem 2 del MGH-SFQ) con otro grupo que no presentaba dificultades (puntuación de 4, es decir, normal, en el ítem 2 del MGH-SFQ), siguiendo indicaciones del punto de corte de estudios previos (Sierra et al., 2012). Para evitar errores tipo I, en las comparaciones post hoc, se consideró la corrección Bonferroni, por lo que, considerando las comparaciones entre grupos, se estableció un nivel de significatividad de $p<.008$. Este análisis fue complementado con un análisis de la curva ROC (Receiver Operating Characteristic Curve). Es necesario indicar que, al encontrarnos ante una muestra de personas sanas -solo fueron incluidas las que no tenían enfermedades ni presencia de otras patologías-, las puntuaciones obtenidas en la 
Tabla 5. Diferencias entre participantes con dificultades en el funcionamiento sexual relacionado con la excitación y participantes sin dificultades

\begin{tabular}{lccccc}
\hline & \multicolumn{1}{c}{ Grupo } & $M$ & $D T$ & $t$ & $d$ \\
\hline Mujeres & Sin dificultades & 15.54 & 3.22 & & \\
SES & Con dificultades & 14.94 & 2.99 & 0.69 & \\
& Sin dificultades & 7.88 & 1.94 & -2.11 & \\
SIS1 & Con dificultades & 9.00 & 1.83 & & \\
& Sin dificultades & 11.91 & 2.32 & -0.45 & \\
SIS2 & Con dificultades & 12.19 & 1.97 & & \\
Hombres & Sin dificultades & 16.38 & 3.09 & 0.30 & \\
SES & Con dificultades & 16.09 & 2.17 & & \\
& Sin dificultades & 7.67 & 1.96 & $-3.58^{* * *}$ & -1.28 \\
SIS1 & Con dificultades & 9.91 & 1.51 & & \\
SIS2 & Sin dificultades & 10.37 & 2.31 & -0.69 & \\
& Con dificultades & 10.91 & 2.66 & & \\
\hline
\end{tabular}

Nota. SES: excitación sexual; SIS1: inhibición sexual por miedo al fallo en el rendimiento/ejecución sexual; SIS2: inhibición sexual por miedo a las consecuencias de la ejecución sexual. $* * * p<.001$.

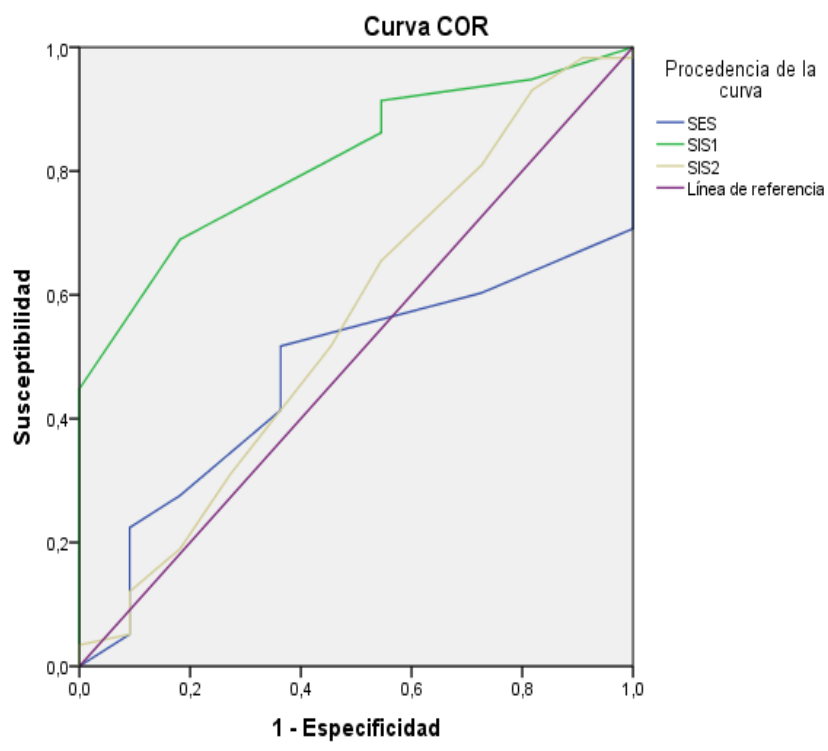

Figura 1. Curva ROC de SES, SIS1 y SIS2 en hombres

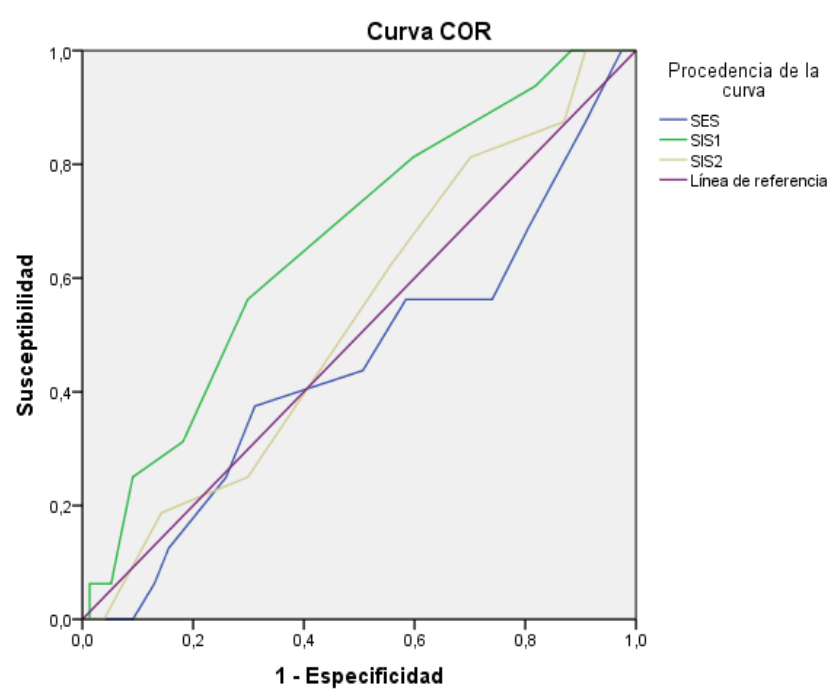

escala MGH-SFQ oscilaron entre 2 y 4, no existiendo respuestas que indicasen una disminución "total" o "marcada" de su funcionamiento sexual. Tal como se observa en la Tabla 5, únicamente hubo diferencias estadísticamente significativas en la dimensión SIS1 en hombres, siendo las puntuaciones mayores en los participantes con peor funcionamiento de la respuesta sexual. De modo similar, en el análisis de la curva COR, representado en las Figuras 1 y 2, se observó la capacidad predictiva de SIS1 en hombres y mujeres, respectivamente. En concreto, en los hombres se observó una probabilidad de clasificación correcta del $81.7 \%$ de los casos ( $p=.001)$, con un intervalo de confianza asintótico al $95 \%$ de límite inferior igual a .70 y superior de .92. El índice de Youden indicó que a partir de puntuaciones directas de 8.50 en SIS1, con una sensibilidad del $56 \%$ y especificidad del $30 \%$, se determina riesgo de dificultades en el funcionamiento sexual. En mujeres, se observó una probabilidad de clasificación correcta del 33\% de los casos $(p=.04)$, con un intervalo de confianza asintótico al 95\% de límite inferior igual a .19 y superior de .47. El índice de Youden indicó, como punto de corte la puntuación directa de 8.50 o superiores como indicador de dificultad en funcionamiento sexual, con una sensibilidad y especificidad del $31 \%$ y $80 \%$ respectivamente.

Figura 2. Curva ROC de SES, SIS1 y SIS2 en mujeres 


\section{Discusión}

El Modelo de Control Dual (MCD) se ha utilizado para explicar, entre otros comportamientos sexuales, las disfunciones sexuales (Bancroft, Carnes et al., 2005; Bancroft, Herbenick et al., 2005; Bancroft \& Janssen, 2000, 2001; Janssen et al., 2002a, 2002b; Moyano \& Sierra, 2014; Sanders, Graham, \& Milhausen, 2008; Santos-Iglesias et al., 2017; Velten et al., 2017). Según el MCD, una persona que puntúa alto en inhibición sexual y bajo en excitación sexual puede tener dificultad para excitarse sexualmente y podría estar predispuesta a sufrir disfunciones sexuales (Bancroft \& Janssen, 2000, 2001).

Las escalas SIS/SES-SF (Carpenter et al., 2011), adaptadas a población española por Moyano y Sierra (2014), son un instrumento breve que evalúa los componentes del $\mathrm{MCD}$, tanto en hombres como en mujeres y que, en el caso de España, sus puntuaciones son comparables entre ambos sexos porque su estructura ha demostrado ser invariante en cuanto al sexo (Moyano \& Sierra, 2014). Con el objetivo de aportar indicios de validez a sus medidas se diseñó este estudio, para examinar su capacidad para (a) predecir la excitación sexual subjetiva y fisiológica en una situación concreta, y (b) distinguir entre personas con y sin dificultades en la dimensión de excitación de la respuesta sexual.

La propensión a excitarse (SES) planteada desde el MCD se ha asociado a una mayor excitación sexual subjetiva y a mayor intensidad de respuesta genital ante estímulos visuales (Granados et al., 2018; Hodgson, 2013; Hodgson et al., 2016; Janssen et al., 2002b), mientras que la propensión a inhibirse (SIS) lo ha hecho con una menor excitación sexual subjetiva y objetiva (Hodgson et al., 2016). En el presente estudio, únicamente se encontró en las mujeres una asociación entre SES y excitación sexual subjetiva. La respuesta genital no se asoció con ninguna de las dimensiones de las SIS/SES-SF. Es decir, en las mujeres, la propensión a excitarse sexualmente se asocia al nivel de excitación subjetiva informada ante el visionado de estímulos eróticos, pero no se relaciona con la valoración de las reacciones genitales que se producen ante dichos estímulos. Los resultados obtenidos en las mujeres apuntan que las dimensiones excitación/inhibición sexuales concuerdan más con las respuestas sexuales subjetivas que con las fisiológicas, lo que podría ser debido a que la excitación sexual genital sigue un patrón inconsciente mientras que las sensaciones sexuales subjetivas, valoradas mediante las escalas VES y VSG, siguen un procesamiento consciente (Janssen \& Everaerd, 1993; Janssen, Everaerd, Spiering, \& Janssen, 2000; Laan et al., 1995; Laan \& Janssen, 2007). En los hombres, la ausencia de relación de la propensión a excitarse/inhibirse sexualmente con las medidas subjetiva y fisiológica de la excitación sexual podría ser consecuencia de que la variabilidad de sus puntuaciones en ambas medidas fuese limitada. Janssen et al. (2002b), al comparar grupos de hombres con altas y bajas puntuaciones en SES, SIS1 y SIS2 ante estímulos sexuales visuales, observaron que aquellos con altas puntuaciones en SES y bajas puntuaciones en SIS2 también tenían una mayor respuesta eréctil, no encontrando ningún resultado significativo con SIS1; asimismo, una mayor excitación sexual subjetiva fue asociada a altas puntuaciones en SES, no obteniendo resultados significativos con las dimensiones de SIS.

La falta de correlación entre las dimensiones de inhibición sexual y la excitación sexual subjetiva puede deberse, al menos en el caso de las mujeres, a que las escalas SIS/SES-SF no estén recogiendo matices característicos de la inhibición sexual femenina. De hecho, Granados et al. (2018), empleando la Sexual Excitation/Sexual Inhibition Inventory for Women (SESII-W; Graham, Sanders, \& Milhausen, 2006; Granados, Salinas, \& Sierra, 2017), encontraron una correlación moderada entre la inhibición sexual y la excitación sexual autoinformada, jugando un papel importante en esta asociación aspectos como la importancia de la relación de pareja, que no se recogen en las SIS/SES-SF (e. g., "Si pienso que mi pareja puede herirme emocionalmente, me cohíbo o freno sexualmente" o "Sería para mí difícil excitarme sexualmente con alguien que tiene una relación con otra persona"). Del mismo modo, Velten, Scholten, Graham, Adolph y Margraf (2016) observaron que algunos factores de inhibición sexual del SESII-W predecían la concordancia sexual 
(relación entre la respuesta subjetiva y genital). De la misma forma en hombres, el contenido de los ítems de los dos factores de inhibición puede no estar representado en los ítems de VES y VSG, ya que estos últimos solo mencionan aspectos de la excitación sexual obviando el componente inhibitorio.

El hecho de que la excitación sexual subjetiva y objetiva no sigan en las mujeres patrones similares en su asociación con la propensión a excitarse puede deberse a la discordancia entre ambas medidas encontrada en estudios previos (Chivers, Seto, Lalumière, Laan, \& Grimbos, 2010; Sierra et al., 2018). Es posible que la experiencia de excitación sexual en las mujeres esté más determinada por el estado de excitación general que por la respuesta genital en particular (Graham, Sanders, Milhausen, \& McBride, 2004). A pesar de esta discrepancia entre ambas medidas, se esperaba que, las dimensiones del MCD se relacionasen con la excitación sexual objetiva y subjetiva, siguiendo probablemente patrones de asociación no similares en cuanto a su intensidad. Sin embargo, los resultados indicaron patrones totalmente diferentes. Esta discordancia entre las medidas objetivas y subjetivas, independientemente de las causas que la provoquen, plantean la necesidad de emplear ambas a la hora de analizar la excitación sexual, poniendo en valor la evaluación psicofisiológica de la respuesta sexual.

Por otro lado, se comprobó la capacidad de las escalas SIS/SES-SF para diferenciar a personas con y sin dificultades de excitación en la respuesta sexual. Bancroft (1999) y Bancroft y Janssen (2000) teorizan que la propensión hacia la excitación e inhibición podrían ser determinantes del funcionamiento sexual. En el presente estudio, la dimensión de inhibición sexual relacionada con el miedo a la ejecución sexual (SIS1) se asoció de manera significativa al funcionamiento sexual en los hombres, de manera que aquellos con mayor inhibición de este tipo tienen más dificultades para excitarse durante las actividades sexuales, resultados muy similares a los señalados por otros autores (Moyano \& Sierra, 2014; Santos-Iglesias et al., 2017). La focalización en el rendimiento sexual, que caracteriza a SIS1, podría dar lugar a la ansiedad de ejecución que suele desencadenar la aparición de algunas disfunciones sexuales
(Dove \& Wiederman, 2000; McCabe, 2005). En cuanto a la capacidad diagnóstica de la escala, resulta de interés valorar que, tanto en hombres como en mujeres, la única variable que permite realizar predicciones en cuanto a las dificultades en el funcionamiento sexual sería SIS1, siendo mayor la capacidad de clasificación en ellos que en ellas $(81.7 \%$ vs. $33 \%)$ a partir de puntuaciones superiores a 8.50 . Estudios previos realizados en España muestran la mayor relación entre SIS1 con el funcionamiento sexual (Moyano \& Sierra, 2014), subrayándose de este modo lo apuntado por Bancroft y Janssen (2000) de que SIS1 constituye un "rasgo".

Por último, se deben señalar algunas limitaciones del estudio, como la imposibilidad de generalizar los resultados al tratarse de una muestra incidental formada por jóvenes heterosexuales con un corto rango de edad, debido al interés por controlar el efecto de la edad sobre la respuesta sexual. Futuros estudios deberían incluir participantes con disfunciones sexuales con el fin de consolidar su asociación con SIS1. Asimismo, futuras investigaciones deberían revisar los factores SIS1 y SIS2 por su baja fiabilidad, planteando por ejemplo, la inclusión de ítems más representativos de la inhibición sexual en ambos sexos. Se puede concluir que este estudio avala únicamente en las mujeres la asociación entre la propensión a excitarse sexualmente, medida con las escalas SIS/SES-SF, y la excitación sexual subjetiva experimentada en una situación sexual determinada. Sin embargo, no no se puede concluir que la versión española de estas escalas tenga capacidad para predecir la excitación sexual objetiva, es decir, las reacciones genitales ante estímulos sexuales. Por su parte, la propensión a inhibirse sexualmente, en concreto la asociada al miedo al fallo en el rendimiento sexual, se asocia al funcionamiento sexual, mostrando capacidad para clasificar a hombres y mujeres con y sin dificultades en la fase de excitación de la respuesta sexual.

\section{Referencias}

Bancroft, J. (1999). Central inhibition of sexual response in the male: A theoretical perspective. Neuroscience \& Behavioral Reviews, 23, 763-784. 
doi:10.1016/S0149-7634(99)00019-6

Bancroft, J., Carnes, L., Janssen, E., Goodrich, D., \& Long, J. S. (2005). Erectile and ejaculatory problems in gay and heterosexual men. Archives of Sexual Behavior, 34, 285-297. doi:10.1007/s10508-005-3117-7

Bancroft, J., Graham, C. A., Janssen, E., \& Sanders, S. A. (2009). The Dual Control Model: Current status and future directions. Journal of Sex Research, 46, 121-142. doi:10.1080/00224490902747222

Bancroft, J., Herbenick, D., Barnes, T., HallamJones, R., Wylie, K., Janssen, E., \& Members of BASRT. (2005). The relevance of the dual control model to male sexual dysfunction: The Kinsey Institute/BASRT collaborative project. Sexual and Relationship Therapy, 20, 13-30. doi:10.1080/14681990512331298275

Bancroft, J., \& Janssen, E. (2000). The dual control model of male sexual response: A theoretical approach to centrally mediated erectile dysfunction. Neuroscience \& Biobehavioral Review, 24, 571-579. doi:10.1016/S0149-7634(00)00024-5

Bancroft, J., \& Janssen, E. (2001). Psychogenic erectile dysfunction in the era of pharmacotherapy: A theoretical approach. En J. Mulcahy (Ed.), Male sexual function: A guide to clinical management (pp. 79-89). Totowa, NJ: Humana Press.

Bradford, A., \& Meston, C. M. (2006). The impact of anxiety on sexual arousal in women. Behaviour Research and Therapy, 44, 10671077. doi:10.1016/j.brat.2005.08.006

Carpenter, D. L., Janssen, E., Graham, C. A., Vorst, H., \& Wicherts, J. (2008). Women's scores on the Sexual Inhibition/Sexual Excitation Scales (SIS/SES): Gender similarities and differences. Journal of Sex Research, 45, 36-48. doi:10.1080/00224490701808076

Carpenter, D. L., Janssen, E., Graham, C. A., Vorst, H., \& Wicherts, J. (2011). The Sexual Inhibition/Sexual Excitation Scales-Short Form. En T. D. Fisher, C. M. Davis, W. L. Yarber y S. L. Davis (Eds.), Handbook of sexuality-related measures (pp. 236-239). Nueva York, NY: Routledge.

Chivers, M. L., Seto, M. C., Lalumière, M. L., Laan, E., \& Grimbos, T. (2010). Agreement of self-reported and genital measures of sexual arousal in men and women: A metaanalysis. Archives of Sexual Behavior, 39, 556. doi:10.1007/s10508-009-9556-9

Dove, N. L., \& Wiederman, M. W. (2000). Cognitive distraction and women's sexual functioning. Journal of Sex \& Marital Therapy, 26, 67-78. doi:10.1080/009262300278650

Fava, M., Rankin, M. A., Alpert, L. E., Nierenberg, A. A., \& Worthington, J. J. (1998). An open trial of oral sildenafil in antidepressant-induced sexual dysfunction. Psychotherapy and Psychosomatics, 67, 328331. doi:10.1159/000012299

Graham, C. A., Sanders, S. A., \& Milhausen, R. R. (2006). The Sexual Excitation/Sexual Inhibition Inventory for Women: Psychometric properties. Archives of Sexual Behavior, 35, 397-409. doi:10.1007/s10508-006-9041-7

Graham, C. A., Sanders, S. A., Milhausen, R. R., \& McBride, K. R. (2004). Turning on and turning off: A focus group study of the factors that affect women's sexual arousal. Archives of Sexual Behavior, 33, 527-538. doi:10.1023/B:ASEB.0000044737.62561.fd

Granados, M. R., Carvalho, J., \& Sierra, J. C. (2018). Relationship between sexual excitation/inhibition dimensions and subjective sexual arousal and genital response: The dual control model in women. Manuscrito sometido a publicación.

Granados, M. R., Salinas, J. M., \& Sierra, J. C. (2017). Spanish version of the Sexual Excitation/Sexual Inhibition Inventory for Women: Factorial structure, reliability and validity evidence. International Journal of Clinical and Health Psychology, 17, 65-76. doi:10.1016/j.ijchp.2016.09.003

Gregory, R., Cheng, H., Rupp, H. A., Sengelaub, D. R., \& Heiman, J. R. (2015). Oxytocin increases VTA activation to infant and sexual stimuli in nulliparous and postpartum women. Hormones and Behavior, 69, 82-88. doi:10.1016/j.yhbeh.2014.12.009

Hodgson, B. (2013). Using the Dual Control Model to investigate the relationship between mood, physiological and self-reported sexual arousal in men and women (Tesis doctoral). 
Universidad Guelph, Guelph, Ontario, Canadá.

Hodgson, B., Kukkonen, T. M., Binik, Y. M., \& Carrier, S. (2016). Using the Dual Control Model to investigate the relationship between mood, genital, and self-reported sexual arousal in men and women. Journal of Sex Research, 53, 979-993. doi:10.1080/00224499.2015.1110107

Janssen, E., \& Bancroft, J. (2007). The DualControl Model: The role of sexual inhibition and excitation in sexual arousal and behavior. In E. Janssen (Ed.), The Psychophysiology of Sex (pp. 197-222). Bloomington, IN: Indiana University Press.

Janssen, E., \& Everaerd, W. (1993). Determinants of male sexual arousal. Annual Review of Sex Research, 4, 211-245. doi:10.1080/10532528.1993.10559888

Janssen, E., Everaerd, W., Spiering, M., \& Janssen, J. (2000). Automatic processes and the appraisal of sexual stimuli: Toward an information processing model of sexual arousal. Journal of Sex Research, 37, 8-23. doi:10.1080/00224490009552016

Janssen, E., Vissenberg, M., Visser, S., \& Everaerd, W. (1997). An in vivo comparison of two circumferential penile strain gauges: Introducing a new calibration method. Psychophysiology, $\quad 34, \quad$ 717-720. doi:10.1111/j.1469-8986.1997.tb02147.x

Janssen, E., Vorst, H., Finn, P., \& Bancroft, J. (2002a). The Sexual Inhibition (SIS) and Sexual Excitation (SES) scales: I. Measuring sexual inhibition and excitation proneness in men. Journal of Sex Research, 39, 114-126. doi:10.1080/00224490209552130

Janssen, E., Vorst, H., Finn, P., \& Bancroft, J. (2002b). The Sexual Inhibition (SIS) and sexual Excitation (SES) Scales: II. Predicting psychophysiological response patterns. Journal of Sex Research, 39, 127-132. doi:10.1080/00224490209552131

Laan, E., Everaerd, W., \& Evers, A. (1995). Assessment of female sexual arousal: Response specificity and construct validity. Psychophysiology, 32, 476-485. doi:10.1111/j.1469-8986.1995.tb02099.x

Laan, E., \& Janssen, E. (2007). How do men and women feel? Determinants of subjective experience of sexual arousal. En E. Janssen (Ed.), The psychophysiology of sex (pp. 278290). Bloomington, IN: Indiana University Press.

Landry, S. (2016). The impact of sexual attitudes, guilt, knowledge, and propensity for sexual arousal on self-reported sexual response to a sexually explicit stimulus (Tesis doctoral). Universidad Guelph, Guelph, Ontario, Canadá.

Latorre Román, P. A., López Munera R., Izquierdo Rus, T., \& García Pinillos, F. (2018). La satisfacción corporal en adultos españoles, influencia del sexo, edad y estado ponderal. Revista Iberoamericana de Diagnóstico y Evaluación - e Avaliação Psicológica, 47, 83-94. doi:10.21865/RIDEP47.2.06 López Huerta, J. A., González Romo, R. A., \& Tejada Tayabas, J. M. (2017). Propiedades psicométricas de la versión en español de la Escala de Calidad de Vida WHO QoL BREF en una muestra de adultos mexicanos. Revista Iberoamericana de Diagnóstico y Evaluación - e Avaliação Psicológica, 44, 105-115. doi:10.21865/RIDEP44.2.09

Macapagal, K. R., Janssen, E., Fridberg, D. J., Finn, P. R., \& Heiman, J. R. (2011). The effects of impulsivity, sexual arousability, and abstract intellectual ability on men's and women's go/no-go task performance. Archives of Sexual Behavior, 40, 995-1006. doi:10.1007/s10508-010-9676-2

McCabe, M. P. (2005). The role of performance anxiety in the development and maintenance of sexual dysfunction in men and women. International Journal of Stress Management, 12, 379-388. doi:10.1037/1072- 5245.12.4.379

Mosher, D. L. (2011). Multiple indicators of subjective sexual arousal. En T. D. Fisher, C. M. Davis, W. L. Yarber y S. L. Davis (Eds.), Handbook of sexuality-related measures (pp. 59-61). Nueva York, NY: Routledge.

Moyano, N., \& Sierra, J. C. (2014). Validación de las Escalas de Inhibición Sexual/Excitación Sexual-Forma Breve (SIS/SES-SF). Terapia Psicológica, 32, 87-100. doi:10.4067/S0718-48082014000200002

Prause, N., Janssen, E., \& Hetrick, W. P. (2008). Attention and emotional responses to sexual stimuli and their relationship to sexual desire. 
Archives of Sexual Behavior, 37, 934-949. doi:10.1007/s10508-007-9236-6

Rupp, H. A., James, T. W., Ketterson, E. D., Sengelaub, D. R., Janssen, E., \& Heiman, J. R. (2009). Neural activation in the orbitofrontal cortex in response to male faces increases during the follicular phase. Hormones and Behavior, 56, 66-72. doi:10.1016/j.yhbeh.2009.03.005

Sanders, S. A., Graham, C. A., \& Milhausen, R. R. (2008). Predicting sexual problems in women: The relevance of sexual excitation and sexual inhibition. Archives of Sexual Behavior, 37, 241-251. doi:10.1007/s10508-007-9235-7

Santos-Iglesias, P., Castro, A., Barbosa, M., \& Nobre, P. (2017). Sexual functioning in Spanish and Portuguese young adults: Initial validation of the Arizona Sexual Experience Scale. International Journal of Sexual Health, 29, 155-167. doi:10.1080/19317611.2016.1259706

Sierra, J. C., Álvarez-Muelas, A., Arcos-Romero, A., Calvillo, C., Torres-Obregón, R., \& Granados, R. (2018). Relación entre la excitación sexual subjetiva y la respuesta genital: Diferencias entre hombres y mujeres. Revista Internacional de Andrología. Publicación anticipada en línea. doi:10.1016/j.androl.2017.12.004

Sierra, J. C., Arcos-Romero, A. I., Granados, M. R., Sánchez-Fuentes, M. M., Calvillo, C., \& Moyano, N. (2017). Escalas de Valoración de Excitación Sexual y Valoración de Sensaciones Genitales: Propiedades psicométricas en muestras españolas. Revista Internacional de Andrología, 15, 99-107. doi:10.1016/j.androl.2016.10.008

Sierra, J. C., Granados, R., Sánchez-Fuentes, M. M., Moyano, N., \& López, C. (2015, julio). Activación sexual ante estímulos sexuales visuales: Comparación entre hombres $y$ mujeres. Póster presentado en el XXXV Congreso Interamericano de Psicología, Lima, Perú.

Sierra, J. C., Vallejo-Medina, P., Santos-Iglesias, P., \& Lameiras Fernández, M. (2012). Validación del Massachusetts General Hospital-Sexual Functioning Questionnaire (MGH-SFQ) en población española. Atención
Primaria,

44 ,

$516-526$.

doi:10.1016/j.aprim.2012.02.004

Sintchak, G, \& Geer, J. H. (1975). A vaginal plethysmograph system. Psychophysiology, 12, 113-115. doi:10.1111/j.1469-8986.1975.b03074.x

Suschinsky, K. D., Bossio, J. A., \& Chivers, M. L. (2014). Women's genital sexual arousal to oral versus penetrative heterosexual sex varies with menstrual cycle phase at first exposure. Hormones and Behavior, 65, 319-327. doi:10.1016/j.yhbeh.2014.01.006

Vallejo-Medina, P., Pérez-Durán, C., \& SaavedraRoa, A. (2018). Translation, adaptation, and preliminary validation of the Female Sexual Function Index into Spanish (Colombia). Archives of Sexual Behavior, 47, 797-810. doi:10.1007/s10508-017-0976-7

Velten, J., Scholten, S., Graham, C. A., Adolph, D., \& Margraf, J. (2016). Investigating female sexual concordance: Do sexual excitation and sexual inhibition moderate the agreement of genital and subjective sexual arousal in women? Archives of Sexual Behavior, 45, 1957-1971. doi:10.1007/s10508-016-0774-7

Velten, J., Scholten, S., Graham, C. A., \& Margraf, J. (2017). Sexual excitation and sexual inhibition as predictors of sexual function in women: A cross-sectional and longitudinal study. Journal of Sex and Marital Therapy, 43, 95-109. doi:10.1080/0092623X.2015.1115792 